\title{
Potencialidad de Cedrela odorata (Meliaceae) para estudios dendrocronológicos en la selva central del Perú
}

\author{
Manuel Jesús Pereyra Espinoza ${ }^{1}$, Gaby Janet Inga Guillen ${ }^{1}$, Mariano Santos Morales $^{2}$ \& \\ Rodolfo Rodríguez Arisméndiz ${ }^{3}$ \\ 1. Facultad de Ciencias Forestales y del Ambiente, Universidad Nacional del Centro del Perú, Av. Mariscal Ramón \\ Castilla km cinco No 3809, El Tambo, Huancayo, Perú; manuelpereyraespinoza@yahoo.es, \\ gabyingaguillen@yahoo.es \\ 2. Instituto Argentino de Nivología, Glaciología y Ciencias Ambientales CCT-CONICET, Mendoza, Argentina; \\ mmorales@mendoza-conicet.gob.ar \\ 3. Facultad de Ingeniería, Universidad de Piura, Apartado 353, Piura, Perú; rodolfo.rodriguez@udep.pe
}

Recibido 12-XI-2012. Corregido 30-III-2013. Aceptado 30-IV-2013.

\begin{abstract}
Cedrela odorata (Meliaceae) potential for dendrochronological studies in the Selva Central of Perú. Despite the progress made during the past 20 years, searching dendrochronological potential in tropical and subtropical tree species, tropical dendrochronology, is still in a development stage. The aim of this research was to determine the potential of $C$. odorata for dendrochronological studies in the Selva Central of Perú. The tree-ring anatomical characteristics were carefully examined and we were able to develop a 215 year (1 795-2 009) tree-ring chronology and correlate it with precipitation records. The tree-ring chronology was developed based on 47 series of 27 trees. Tree rings are clearly delimited by large pore diameters in earlywood and small ones in latewood associated with marginal and paratracheal parenchyma. The tree-ring chronology was related to precipitation records from Satipo and significant correlations were found with the previous rainy season and late dry season of the current growth period. Moreover, we found close relationship between tree growth and total precipitations of the hydrological period (December to September) for the interval 1 990-2 009. These results demonstrate the influence of rainfall at different stages of $C$. odorata radial growth. The good discrimination of annual rings, strong relationship with precipitation, the wide range and longevity of trees (200 years) make $C$. odorata a very promising species for dendrochronological studies in tropical and subtropical forest of America. Rev. Biol. Trop. 62 (2): 783-793. Epub 2014 June 01.
\end{abstract}

Key words: annual tree rings, tropical trees, growth-climate relationship, wood anatomy, tropical dendrochronology.

La dendrocronología es la ciencia que estudia los anillos de crecimiento de los árboles para reconstruir, con resolución anual, eventos climáticos y procesos ecológicos pasados (Fritts, 1976). Las técnicas dendroclimatológicas se aplican en especies que presenten anillos anuales bien diferenciados y un crecimiento radial influenciado por las fluctuaciones climáticas (Fritts, 1976).

La presencia de anillos de crecimiento anuales visibles es propia de la mayoría de especies arbóreas y arbustivas que se desarrollan en regiones templadas y frías donde existe una fuerte estacionalidad climática responsable de causar un periodo de dormancia o reposo en el crecimiento. Por el contrario, las regiones tropicales y subtropicales han sido tradicionalmente consideradas como regiones poco adecuadas para aplicar las técnicas dendrocronológicas tradicionales, debido a que en muchos casos los anillos de crecimiento no son claramente distinguibles en comparación con las especies de climas templados (Worbes, 2002), o por la falta de periodicidad en la formación anual de los anillos (Priya \& Bhat, 1998). Muchas de las especies tropicales 
presentan, además, una corta longevidad y en general, una baja preservación de la madera muerta (Boninsegna \& Villalba, 1996; Worbes, 1999; Villalba, 2000), que no permiten desarrollar cronologías de largo plazo, que superen al menos, el período cubierto por datos instrumentales de precipitación y temperatura. Sin embargo, en determinadas regiones tropicales, la existencia de cierta estacionalidad climática, junto con las condiciones particulares del sitio, inducen períodos de reposo invernal y la demarcación de las bandas de crecimiento. (Morales, et al. 2001; Roig, 2000; Schöngart, Piedade, Ludwigshausen \& Worbes, 2002; Worbes, 1995, 1999). A partir de los trabajos pioneros de Vetter \& Botosso (1989) y Worbes (1985, 1989), el número de estudios sobre anillos de árboles en la región tropical y subtropical de Sudamérica, ha aumentado de manera exponencial a medida que se ha incrementado la lista de especies con anillos de crecimiento anuales claramente identificables. Entre tanto, la mayoría de estos estudios se refieren a la presencia y la periodicidad de los anillos de crecimiento (Oliveira, 2007; Oliveira, Santarosa, Pillar \& Roig, 2009; Worbes, 2002), mientras que aún siguen siendo escasos los estudios donde se desarrollen cronologías de ancho de anillos y se relacionen con variables climáticas (Baker, Palmer \& D'arrigo, 2008; Brienen \& Zuidema, 2005b; Dünisch, Montoia \& Bauch, 2003; Dünisch, 2005; López \& Villalba, 2011; Morales et al. 2012; Morales, Villalba, Grau \& Paolini, 2004; Ramírez \& Del Valle, 2012); Rodríguez et al. 2005; Schöngart et al. 2004; Solíz et al. 2009; Worbes, 2002).

Aunque menos pronunciados que en las regiones áridas, montañosas o templadas, la región boscosa de la Amazonía oriental y central peruana $\left(1^{\circ}-13^{\circ} \mathrm{S}\right.$ y $\left.70^{\circ}-75^{\circ} \mathrm{W}\right)$ experimenta cambios estacionales en las precipitaciones, concentrándose entre 65 y $75 \%$ de las lluvias durante el período octubre-marzo (estación lluviosa) según las distintas localidades (Servicio Nacional de Meteorología e Hidrología del Perú [SENAMHI], 2010). La estacionalidad existente en la Amazonía central del Perú podría ser suficiente para inducir la dormancia en el crecimiento radial y la formación de anillos anuales en especies arbóreas sensibles a los cambios anuales de la precipitación. Estudios previos demuestran la presencia de anillos de crecimiento visibles en Myrciaria sp., Chorisia sp. y Cedrela sp. (Schwyzer, 1988). Así mismo, Rosero (2009), Campos (2009) y Huamán (2011) identifican periodicidad anual en la formación de los anillos de crecimiento en Swietenia macrophylla $\mathrm{K}$., Cedrelinga cateniformis D. y Hymenaea courbaril L. respectivamente.

Cedrela odorata L., es una de las principales especies arbóreas de las regiones tropicales debido a su importancia maderera y a su amplia distribución, desde Centroamérica hasta la región norte de Argentina $\left(26^{\circ} \mathrm{N}-28^{\circ}\right.$ $\mathrm{S})$. Por tal motivo, la periodicidad anual en la formación de los anillos de crecimiento de $C$. odorata precisa ser evaluada, como así también la respuesta del crecimiento a los cambios climáticos y ecológicos. El objetivo de este estudio fue evaluar el potencial dendrocronológico de $C$. odorata para su posterior uso en estudios ecológicos y climatológicos destinados a reconstruir los cambios ambientales pasados en la región de la Selva Central del Perú. Para lo cual, se describieron en primer término las características anatómicas que definen sus anillos de crecimiento, luego se desarrolló la cronología de ancho de anillos y se establecieron las relaciones entre el crecimiento radial y las fluctuaciones climáticas.

\section{MATERIALES Y MÉTODOS}

Área de estudio: Las muestras dendrocronológicas utilizadas para este estudio las recolectamos en los bosques naturales de la Comunidad Nativa "Tres Unidos de Matereni" $\left(11^{\circ} 45^{\prime} 51^{\prime \prime} \mathrm{S}-74^{\circ} 14^{\prime} 18^{\prime \prime} \mathrm{W}\right)$ a una altitud de $1250 \mathrm{~m}$, distrito de San Martin de Pangoa, provincia de Satipo, Perú. Ecológicamente, el área pertenece a la zona de vida del Bosque Húmedo Premontano Tropical (BHPT), el cual se distribuye entre los 500 y los $2000 \mathrm{~m}$ de altitud. Sin embargo, Reátegui \& Martínez (20011, mencionan que el Cedrela odorata conforma 
el Bosque Húmedo de Montaña alta (BHMa), el cual se caracteriza por presentar un relieve de tipo montañoso con pendientes superiores al $50 \%$. Por ello, este bosque es considerado para fines de protección de suelos, regulador del régimen hídrico, protección de la fauna, banco de germoplasma y como belleza escénica.

Entre las principales especies forestales que comúnmente acompañan a $C$. odorata se encuentran: Cedrelinga catenaeformis, Brosimum alicastrum, Juglans neotropica, Matisia bicolor, Hura crepitans, Guazuma crinita, Brosimum utile, Ficus lauretana y Schizolobium amazonicum. Estas especies conforman el dosel más alto del bosque, alcanzando hasta $35 \mathrm{~m}$. El segundo estrato consta de árboles de $30 \mathrm{~m}$ de altura, mientras que el tercero y cuarto estrato presentan árboles más pequeños, delgados y con deformaciones y alturas entre 10 y $20 \mathrm{~m}$. La vegetación del piso sotobosque es relativamente escasa debido a la fuerte competencia radicular y a la sombra dominante (Oficina Nacional de Evaluación y de Recursos Naturales [ONERN], 1976).

En el área de muestreo, la precipitación aumenta con la altitud, hasta los $1500 \mathrm{~m}$ y por encima de esta altitud decrece. Los datos climáticos provenientes de la estación meteorológica

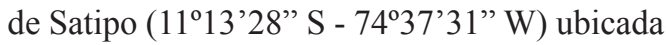
a $660 \mathrm{~m}$ de altitud, podrían considerarse como representativos de las condiciones climáticas que imperan en el sitio de estudio. En Satipo, la temperatura media anual es de $23^{\circ} \mathrm{C}$, con una amplitud térmica entre el mes más fríos y el más cálido menor a $3^{\circ} \mathrm{C}$ (Fig. 1); mientras la precipitación total anual alcanza los $2230 \mathrm{~mm}$ en promedio. En el climograma (Fig. 1), se observa que la temporada de lluvias se inicia en octubre, ascendiendo progresivamente hasta alcanzar el máximo en enero, febrero y marzo, luego decrece paulatinamente hasta llegar al mínimo en junio-julio. La mayor humedad relativa ocurre de setiembre a mayo, coincidiendo con los meses más calurosos mientras que la menor ocurre de junio a agosto, coincidiendo con los meses más fríos (Rodríguez, 2011).

Obtención y procesamiento de muestras: Entre noviembre del 2010 y junio 2011 recolectamos muestras dendrocronológicas de 54 árboles de C. odorata. Debido a las anomalías anatómicas que suelen presentar las especies leñosas que crecen en regiones tropicales y para lograr una definición precisa de la formación anual de los anillos de crecimiento, utilizamos dos métodos de muestreo: 1) El tradicional método dendrocronológico (Stokes \& Smiley, 1968), mediante el cual utilizamos barrenos de incremento para obtener cilindros de madera de aproximadamente $5 \mathrm{~mm}$ de diámetro siguiendo el radio del árbol. La presencia de aletones o contrafuertes es una característica frecuente en

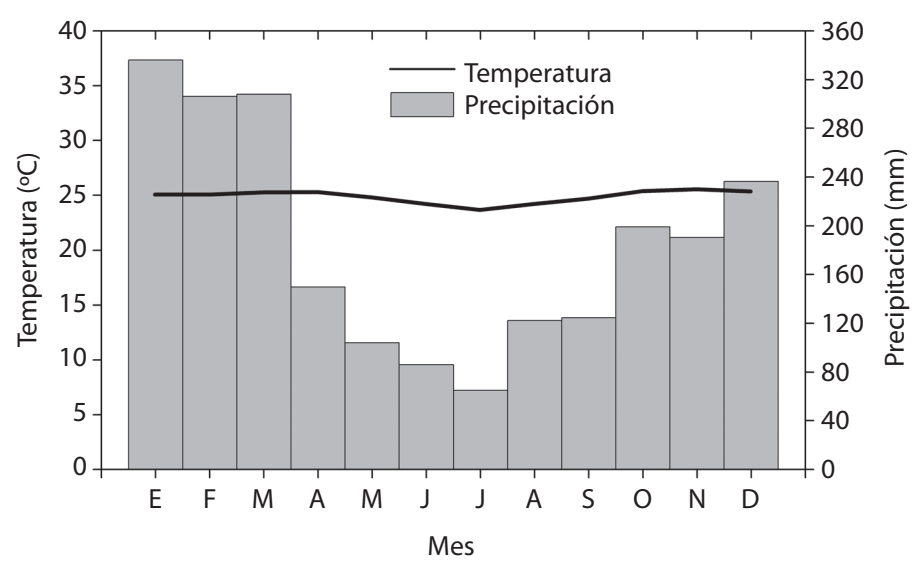

Fig. 1. Climograma correspondiente a la estación meteorológica de Satipo, para el período 1990-2009.

Fig. 1. Climate diagram of Satipo weather station, for the period 1990-2009. 
C. odorata, por lo tanto, la altura de muestreo varió entre $1.30 \mathrm{~m}$ y $3 \mathrm{~m}$, pero siempre por encima de los aletones. Extrajimos al menos dos muestras por árbol y en total recolectamos 78 muestras provenientes de 24 árboles vivos y 12 árboles muertos. 2) El segundo método está basado en la obtención de secciones transversales de árboles utilizados para aprovechamiento forestal (Lopez \& Villalba, 2011; Worbes, 2002). La sección transversal completa del fuste del árbol, brinda mayor campo de observación de la muestra y facilita la identificación de los anillos de crecimiento anuales. Debido a las anomalías anatómicas que suelen presentar las especies leñosas que crecen en regiones tropicales (como por ejemplo la formación de lentes o falsos anillos), por lo general se recomienda trabajar con la sección transversal del fuste en vez de la tradicional muestra de barreno de incremento (López \& Villalba, 2011; Worbes, 2002). Realizamos el muestreo en el aserradero de la empresa NEMATSA S.R.L (carretera marginal kilómetro dos, provincia de Satipo). En total obtuvimos 18 secciones transversales de las primeras trozas forestales de cada árbol.

Todos los árboles muestreados en este estudio provienen de sitios lejanos a los cuerpos de agua, de manera de evitar incluir individuos con provisión continua de agua y que en consecuencia formarían anillos de crecimiento con baja variabilidad interanual, es decir complacientes (Fritts, 1976).

Con el objeto de determinar las características anatómicas del leño, asociados a la delimitación de los anillos de crecimiento, se cortaron cubos de madera de dos centímetros y medio de lado del material colectado. Luego, estos se ablandaron hirviéndolos en agua durante tres horas, para obtener láminas de corte transversal de 30 a $40 \mu$ de espesor mediante un micrótomo Leica modelo SM 2000R de deslizamiento horizontal. Las secciones fueron teñidas con safranina y montadas en bálsamo de Canadá para su posterior observación microscópica (Johansen, 1940). Finalmente, para la descripción anatómica, se ha seguido la metodología de la Asociación
Internacional de los Anatomistas de la Madera (IAWA Committee, 1989).

Las muestras fueron procesadas siguiendo las técnicas convencionales empleadas en dendrocronología (Stokes \& Smiley, 1968). Las muestras de barreno de incremento fueron montadas sobre regletas de madera acanaladas, de aproximadamente un centímetro de espesor por dos centímetros de ancho. Luego, junto con las rodajas, se pulieron con lijas de granos progresivamente más finos. Los anillos de crecimiento fueron correctamente datados al año de formación y medidos con un Equipo Velmex a una precisión de $0.001 \mathrm{~mm}$. La calidad del fechado y de la medición fue controlada utilizando el programa COFECHA (Holmes, 1983), que calcula índices de correlación entre las series individuales de anchos de anillos para identificar anillos ausentes o falsos. Las series de ancho de anillos resultantes de la medición de las bandas de crecimiento, fueron estandarizadas para eliminar las tendencias biológicas propias del crecimiento y minimizar las variaciones en el crecimiento que no son comunes a todos los árboles y que no están relacionadas con las variaciones climáticas (Fritts, 1976). La estandarización permite promediar los anchos de anillos de la cronología, sin que el promedio sea dominado por los individuos de mayor crecimiento. Las series fueron estandarizadas y promediadas para obtener una cronología final de los sitios muestreados empleando el programa ARSTAN (Cook, 1985; versión ARS40Cwin). Este programa genera cronologías combinando las series estandarizadas de ancho de anillos con una media doblemente ponderada (Cook, 1985). La estandarización ajusta las series de ancho de anillos observados a una curva o línea de tendencia y genera un índice adimensional a partir de la división del ancho de anillo observado por el valor esperado. En nuestro caso, el ancho de anillo fue estandarizado empleando un método conservador de estandarización, ajustando una curva exponencial negativa o lineal a cada serie individual. Finalmente, la cronología obtenida constituye una serie temporal que representa las variaciones 
anuales en el crecimiento radial de los árboles de $C$. odorata muestreados.

Análisis de la relación entre el crecimiento anual y las precipitaciones: Con el objetivo de determinar la relación entre el crecimiento radial y el registro instrumental de precipitación de la estación meteorológica de Satipo para el período común 1990-2009, realizamos dos aproximaciones: 1- Utilizamos una función de correlación (Blasing, Solomón \& Duvick, 1984), donde correlacionamos los índices finales de ancho de anillos con los datos de precipitación, tomados mes por mes. Generalmente, como el crecimiento de un año dado puede estar influenciando por condiciones climáticas del año previo, el período de comparación comprendió un periodo de 29 meses, abarcando la estación de crecimiento de dos años previos y del corriente. 2- Comparamos el crecimiento interanual de $C$. odorata con la precipitación total de la estación meteorológica de Satipo, correspondiente al ciclo hidrológico casi anual, que empieza en diciembre del año previo y finaliza en setiembre del año corriente.
Los datos instrumentales de precipitación total mensual y de temperatura media mensual de la estación de Satipo, fueron cedidos por el SENAMHI.

\section{RESULTADOS}

La descripción macroscópica revela que los anillos de crecimiento de Cedrela odorata están formados por madera temprana y madera tardía, la primera generalmente de color más claro que la segunda. Los anillos de crecimiento son distinguibles a simple vista por su porosidad semicircular. La observación microscópica de los cortes histológicos revela que los anillos de crecimiento están delimitados por poros de mayor diámetro en el inicio del periodo vegetativo (madera temprana), dispuestos en hileras tangenciales, en una transición gradual terminando en poros de menor diámetro (madera tardía), los cuales están asociados a bandas de parénquima marginal y paratraqueal (Fig. 2). La banda de tejido parenquimático, formada muy probablemente al final de la estación de crecimiento, se extiende

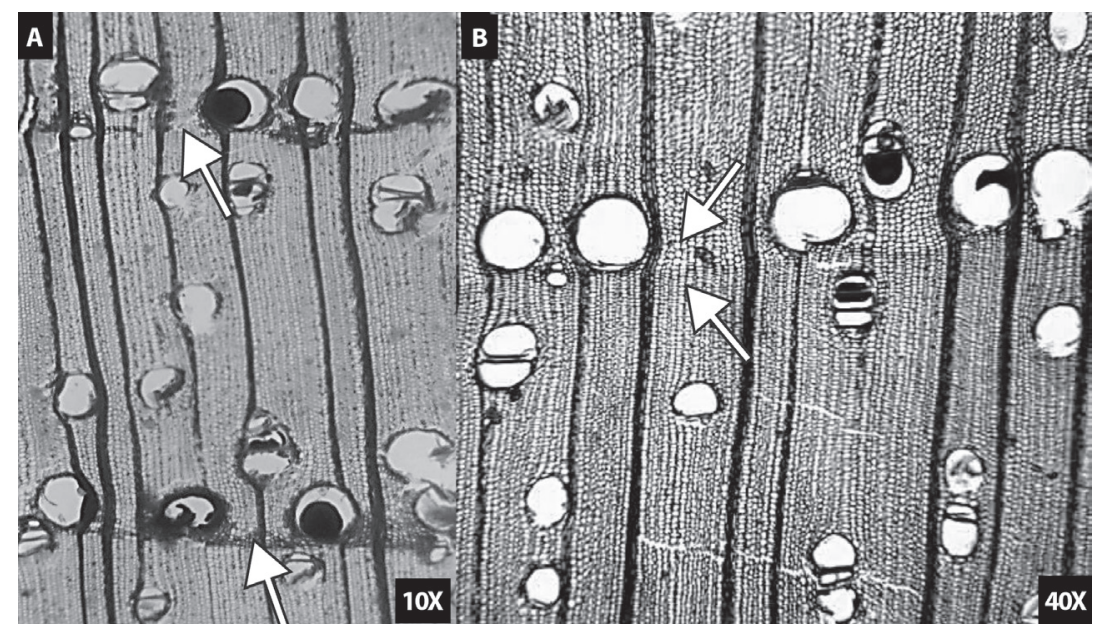

Fig. 2. Anillos de crecimiento en un corte transversal de Cedrela odorata. (A) El límite del anillo está definido por una banda estrecha de parénquima marginal (flechas blancas), poros de mayor tamaño y porosidad semicircular. (B) La diferencia de tamaño de poros revela la transición entre un anillo y el siguiente (flechas blancas). Los cuadros negros indican el aumento microscópico utilizado para describir la anatomía de los anillos de crecimiento. Los cuadros indican el aumento microscópico utilizado para describir la anatomía de los anillos de crecimiento.

Fig. 2. Cross section of Cedrela odorata tree rings. (A) The limit of tree rings (white arrows) is defined by a narrow band of marginal parenchyma and semicircular porosity. (B) The difference of the pore's size highlight the transition (white arrows) between two consecutive growth rings. Black rectangles indicate the microscope magnification used to describe tree growth anatomy. 
en forma ininterrumpida a lo largo del borde del anillo de crecimiento.

De los 54 árboles muestreados, el 50\% (27 árboles) pudieron ser datados correctamente. La cronología final de ancho de anillos está conformada por 47 series de 27 árboles de C. odorata y cubre el periodo 1795-2009 (Fig. 3); sin embargo, recién a partir del año 1850 la cronología está replicada con más de 10 series. Los estadísticos comúnmente usados (Briffa 1995) para establecer la calidad de la cronología (desviación estándar $\mathrm{DE}=0.27$, sensibilidad media $\mathrm{SM}=0.27$, la correlación media entre muestras $\mathrm{RBAR}=0.26$ y EPS $=0.87$ ), indican un alto porcentaje de señal común en las variaciones interanuales del crecimiento radial entre los individuos que conforman la cronología. La función de correlación mostró que el crecimiento radial de $C$. odorata en el sitio de estudio, está fuertemente controlado por las condiciones climáticas correspondiente a los meses de la estación de crecimiento del período previo y comienzos del corriente (Fig. 4). El crecimiento radial se relaciona positivamente con las precipitaciones de diciembre de la estación previa de crecimiento a setiembre del año corriente (Fig. 4), siendo la relación significativa para los meses de enero, febrero, marzo y agosto (Fig. 4).

Con el objeto de determinar la relación interanual entre el crecimiento radial y los registros instrumentales de precipitación para el período común 1990-2 009 (20 años), correlacionamos el índice de ancho de anillos con la precipitación del período correspondiente de diciembre del año previo a setiembre del año corriente, resultando una correlación significativamente positiva $(r=0.69, \mathrm{p}<0.001$, Fig. 5).

\section{DISCUSIÓN}

En este trabajo se presenta la primera cronología de ancho de anillos de 213 años de extensión, desarrollada a partir de Cedrela odorata para la Selva Central del Perú (11\%45'43" $\left.\mathrm{S}-74^{\circ} 14^{\prime} 22^{\prime \prime} \mathrm{O}\right)$. Los resultados de este estudio

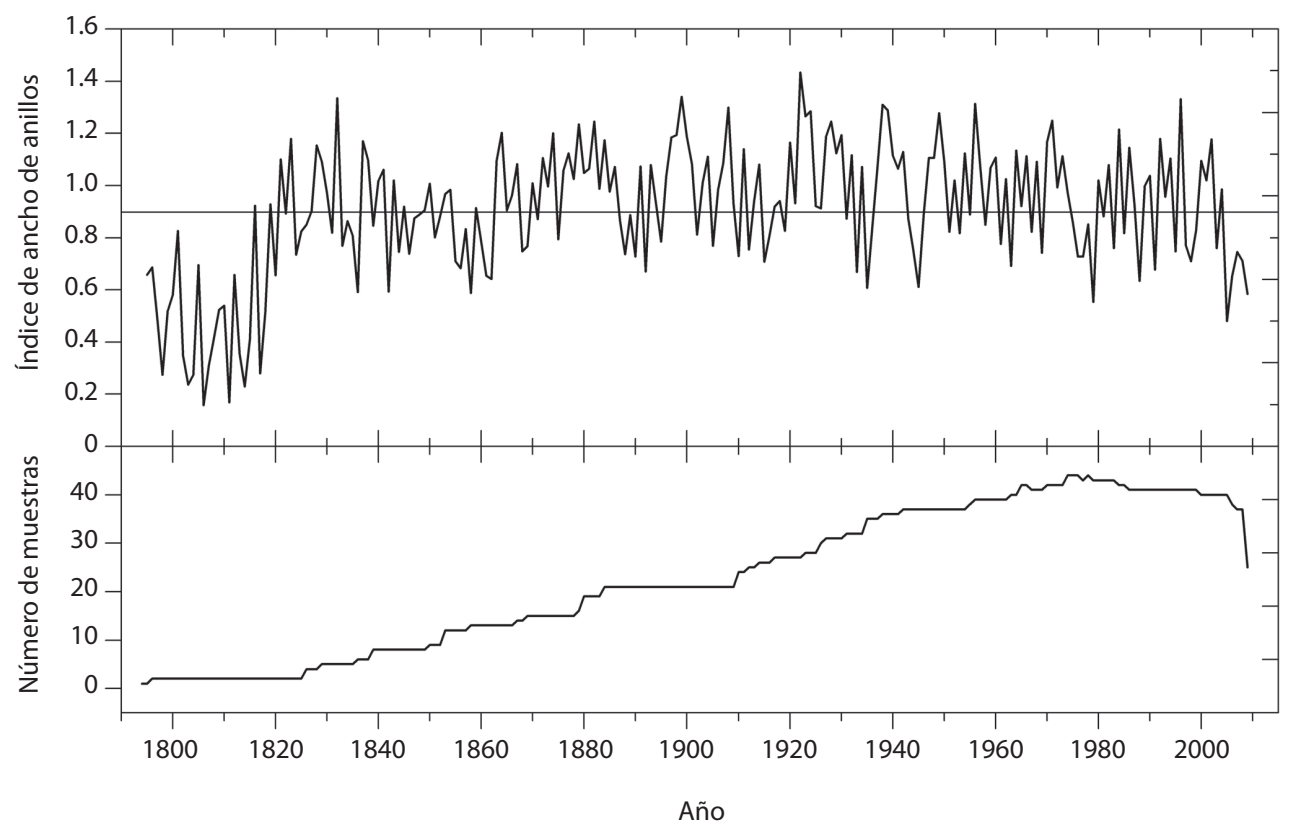

Fig. 3. Cronología de ancho de anillos para Cedrela odorata en la Selva Central del Perú. El número de muestras se indica en la parte inferior.

Fig. 3. Tree-rings chronology from Cedrela odorata at Selva Central of Perú. Sample replication is indicated at the bottom of the figure. 


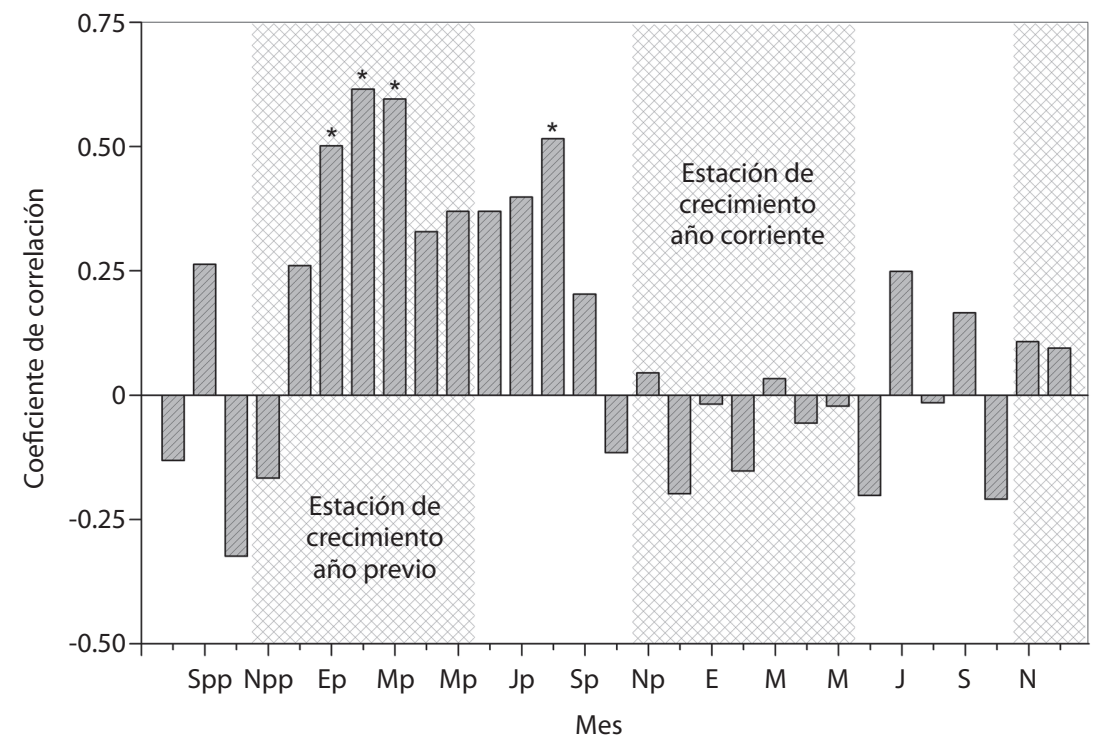

Fig. 4. Función de correlación de la cronología de Cedrela odorata y la precipitación calculada sobre la base de correlaciones entre el ancho de los anillos de crecimiento y las variaciones mensuales en la precipitación de la estación de Satipo para el periodo 1990-2009. (*) Representa la significancia de la correlación con un nivel de confianza del 95\%. (pp) Representa los meses de dos años calendarios previos y (p) a un año calendario previo.

Fig. 4. Correlation function for Cedrela odorata chronology. Monthly precipitation records from Satipo were used for comparison with tree growth over the interval 1990-2009. (*) Represents the 95\% confidence level for the correlation coefficients. (pp) Represents the months of the preceding two calendar years, and (p) of one previous calendar year.

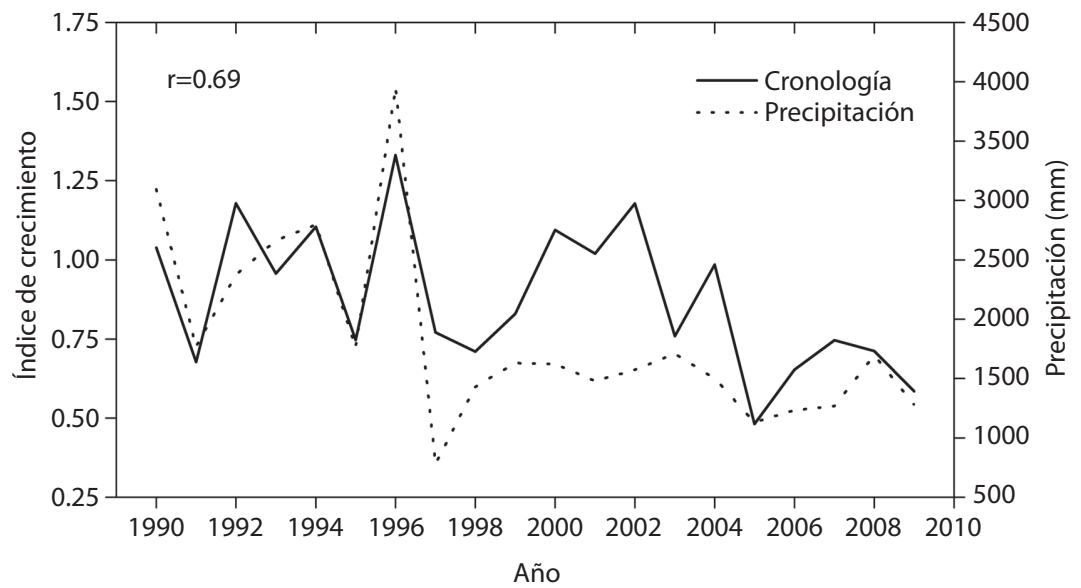

Fig. 5. Correlación entre la cronología final de ancho de anillo de Cedrela odorata y la precipitación total casi anual (diciembre-setiembre) de la estación meteorológica de Satipo.

Fig. 5. Correlation between the Cedrela odorata tree-ring chronology and the total quasi annual precipitation (DecemberSeptember) of the Satipo meteorological station. 
indican que $C$. odorata posee anillos de crecimiento anuales claramente demarcados y que las variaciones en el ancho de los anillos están fuertemente controladas por las características de la lluvias durante la estación de crecimiento previa y comienzos de la corriente.

Es probable que la madera temprana de los anillos de crecimiento de C. odorata, se forme en la temporada de mayor precipitación (octubre-mayo), período en que se observa la brotación de la especie. Completando el anillo con la madera tardía, posiblemente en la temporada de menor precipitación (juniojulio). La presencia de anillos delimitados por poros de mayor diámetro (madera temprana) en el inicio del periodo vegetativo, y poros de menor diámetro (madera tardía) al final del período de crecimiento, asociados a bandas de parénquima marginal y paratraqueal, indican la inducción de un período de dormancia de $C$. odorata como respuesta a cambios estacionales en las precipitaciones. Acevedo \& Kikata (1994), reportan similares resultados basándose en el análisis de cortes histológicos transversales del leño de $C$. odorata en bosques de la Amazonía peruana. Estos autores identificaron anillos de crecimiento definidos por porosidad semicircular y bandas de parénquima marginal. Finalmente, nuestros resultados coinciden con lo reportado por Valencia (2011), quien identificó la presencia de porosidad semicircular en el leño de árboles de C. odorata para la Selva Central del Perú.

Para desarrollar la cronología de ancho de anillos se pudo utilizar el $50 \%$ de los árboles muestreados ( 27 de un total de 54 árboles). Este hecho se debió a que el resto de las series medidas que no integraron la cronología, presentaron anomalías en el crecimiento, tales como: presencia de lentes, anillos indiferenciados y anillos falsos que no permitieron lograr la datación correcta de las muestras. Estos resultados coinciden con estudios previos desarrollados en maderas de diferentes especies tropicales de Perú (Campos, 2009; Huamán, 2011; Rosero, 2009), donde determinan que entre un 35 y $40 \%$ de las muestras obtenidas presentan anomalías en el crecimiento, como tortuosidad, madera de reacción, anillos de crecimiento falsos e indiferenciados.

Debido a la estrecha relación entre la cronología de ancho de anillos y los registros de precipitación, consideramos que las fluctuaciones interanuales de la precipitación son determinantes en el crecimiento radial de los árboles de C. odorata, formando anillos anchos y estrechos en respuesta a una mayor y menor actividad cambial. Estos resultados coinciden con estudios previos desarrollados en regiones tropicales y subtropicales, en donde la disponibilidad de agua tiene gran influencia en el crecimiento de los árboles, no solamente como resultado del déficit de precipitación durante ciertas estaciones del año, sino también debido a la variación de la precipitación de año a año (Brienen, 2005; Brienen \& Zuidema, 2003, 2005a, 2005b; Campos, 2009; Dünisch, Bauch \& Gasparotto, 2002; Dünisch et al. 2003; Huamán, 2011; López \& Villalba, 2011; Morales et al. 2001; Ramírez \& Del Valle, 2012; Roig, 2000; Rosero, 2009; Worbes, 1999, 2001). Nuestros resultados demuestran que el crecimiento de $C$. odorata está influenciado por las precipitaciones de la estación lluviosa previa (principalmente desde enero a marzo) como también por las precipitaciones de finales de la estación seca y comienzos de la época de lluvias (julio-agosto). Similares respuestas fueron encontradas en Juglans australis en las Yungas del noroeste de Argentina (Morales et al. 2004; Villalba, Grau, Boninsegna, Jacoby \& Ripalta, 1998). Mientras que en la Amazonía brasilera, Dünisch et al. (2003) encontró relaciones positivas entre el índice de crecimiento de $C$. odorata y la precipitación para todos los meses analizados, pero solo resultaron significativas durante los meses de la estación de crecimiento previa (marzo, abril, mayo).

Las relaciones positivas obtenidas desde diciembre del período de crecimiento previo a setiembre del año corriente, indican que el máximo crecimiento de $C$. odorata estaría concentrado durante la estación lluviosa y luego entraría en un período de latencia durante la estación seca, mientras que las lluvias a finales 
de la estación seca y comienzo de la temporada de lluvias podrían romper la supuesta latencia.

Por lo tanto, la buena discriminación de los anillos anuales de crecimiento, la fuerte relación con la precipitación, el amplio rango de distribución y la longevidad de los árboles (más de 200 años), hacen de C. odorata una especie promisoria para estudios dendroclimatológicos y dendroecológicos en bosques de la Selva Central del Perú. Estos registros dendrocronológicos podrían proveer de información valiosa sobre las variaciones climáticas de mediano y largo plazo que han influenciado el clima en las regiones tropicales de América. Sin embargo, los estudios dendrocronológicos con $C$. odorata y en general con especies tropicales y subtropicales aún están en una etapa inicial. Numerosos estudios serán necesarios para desarrollar una red de cronologías de ancho de anillos y poder aprovechar en su totalidad el potencial dendrocronológico que nos ofrecen las especies tropicales.

\section{AGRADECIMIENTOS}

Este proyecto fue financiado por el Programa de Ciencia y Tecnología (FINCyT), Proyecto No 067-FINCyT-PIBAP-2008 “Determinación de Incrementos Anuales y Turnos Biológicos de Rotación de Especies Forestales Tropicales para Mejorar el Aprovechamiento de Madera Comercial". Agradecemos a Jimmy Requena, Julio Álvarez y Mauro Rodríguez, por su colaboración en el desarrollo del estudio. A Ana Srur por su asesoramiento. A la empresa NEMATSA S.R.L. por el apoyo logístico para la recolección de muestras dendrocronológicas. Agradecemos a los tres revisores anónimos por sus comentarios detallados que mejoraron considerablemente el manuscrito.

\section{RESUMEN}

En este trabajo determinamos el potencial de Cedrela odorata para estudios dendrocronológicos en la Selva Central del Perú. Para ello, analizamos las características anatómicas que definen los anillos de crecimiento, desarrollamos una cronología de ancho de anillos y relacionamos el crecimiento de los árboles con los registros de precipitación. La cronología de ancho de anillos está compuesta por 47 series de 27 árboles y cubre el período 1795-2009. Los anillos están claramente delimitados por porosidad semicircular, asociados a bandas de parénquima marginal y paratraqueal. El crecimiento de los árboles estuvo relacionado con las precipitaciones de la estación lluviosa previa y con las de finales de la estación seca del corriente período de crecimiento. Así mismo, observamos una estrecha relación entre el crecimiento y las precipitaciones totales, considerando el ciclo hidrológico de diciembre a setiembre, para el periodo 1990-2009. Estos resultados indican la influencia de las precipitaciones en el crecimiento radial de C. odorata. La buena discriminación de los anillos anuales, la fuerte relación con la precipitación, el amplio rango de distribución y la longevidad de los árboles, hacen de $C$. odorata una especie promisoria para estudios dendroclimatológicos y dendroecológicos en los bosques tropicales y subtropicales de América.

Palabras claves: anillos de crecimiento anuales, árboles tropicales, relación clima-crecimiento, anatomía de madera, dendrocronología tropical.

\section{REFERENCIAS}

Acevedo, M., \& Kikata, Y. (1994). Atlas of Peruvian Woods. Lima: Universidad Nacional Agraria la Molina y Universidad de Nagoya.

Baker, P. J., Palmer, J. G., \& D'arrigo, R. (2008). The dendrochronology of Callitris intratropica in northern Australia: annual ring structure, chronology development and climate correlations. Australian Journal of Botany, 56(4), 311-320.

Blasing, T. J., Solomón, A. M., \& Duvick, D. N. (1984). Response functions revisited. Tree-Ring Bulletin, $44,1-15$.

Boninsegna, J. A., \& Villalba, R. (1996). Dendroclimatology in the southern hemisphere: review and prospects. In J. S. Dean, D. M. Meko \& T. W. Swetnam (Eds.), Tree rings, environment and humanity. (pp. 127-141). The University of Arizona, Tucson: Radiocarbon.

Brienen, R .J. W., \& Zuidema, P. A. (2003). Anillos de crecimiento de árboles maderables en Bolivia: su potencial para el manejo de bosques y una guía metodológica. (Informe Técnico $\mathrm{N}^{\mathrm{O}} 7$ ). Riberalta: Programa Manejo de Bosques de la Amazonía boliviana/Instituto de Geologia y Medio Ambiente de la Universidad Mayor de San Andres.

Brienen, R. J. W. (2005). Tree rings in the tropics: a study on growth and ages of Bolivian rain forest trees. (Doctoral dissertation). Universidad de Utrecht, Netherlands.

Brienen, R. J. W., \& Zuidema, P. A. (2005a). Lifetime growth patterns and ages of trees in a tropical forest 
in Bolivia based on tree ring analysis. Journal of Ecology, 94(2), 481-493.

Brienen, R. J. W., \& Zuidema, P. A. (2005b). Relating tree growth to rainfall in Bolivian rain forest: A test for six species using tree ring analysis. Oecologia, 146(1), 1-12.

Briffa, K. R. (1995). Interpreting high-resolution proxy climate data. The example of dendroclimatology. In $\mathrm{H}$. Von Storch \& A. Navarra (Eds.), Analysis of climate variability, applications of statistical techniques. (pp.77-94). Berlin: Springer Verlag.

Campos, L. E. (2009). Dendrocronología en árboles de Tornillo, Cedrelinga cateniformis Ducke (Fabaceae), del Centro de Investigaciones Jenaro Herrera en el noreste de la Amazonia, Región Loreto-Perú. (Tesis de Maestría). Universidad Nacional Agraria de la Molina, Lima.

Cook, E. R. (1985). A time series analysis approach to tree-ring standardization. (Doctoral dissertation). The University of Arizona, Tucson.

Dünisch, O., Bauch, J., \& Gasparotto, L. (2002). Formation of increment zones and intra anual growth dynamics in the xylem of Swietenia macrophylla, Carapa guianensis, and Cedrela odorata (Meliaceae). Iawa Journal, 23(2), 101-119.

Dünisch, O., Montoia, V. R., \& Bauch, J. (2003). Dendroecological investigations on Swietenia macrophylla King and Cedrela odorata L. (Meliaceae) in the central Amazon. Trees, 17(3), 244-250.

Dünisch, O. (2005). Influence of the El-Niño southern oscillation on cambial growth of Cedrela fissilis Vell. in tropical and subtropical Brazil. Journal of Applied Botany and Food Quality, 79(1), 5-11.

Fritts, H. C. (1976). Tree rings and climate. United Kingdom: Academic Press, London.

Holmes, R. (1983). Computer-assisted quality control in tree ring dating and measurement. Tree Ring Bulletin, 43, 69-75.

Huamán, C. I. (2011). Dendrocronología de árboles de azúcar huayo (Hymenaea courbaril L.) en la Región de Madre de Dios (provincia de Tahuamanu)-Perú. (Tesis de Maestría). Universidad Nacional Agraria de la Molina, Lima.

International Association of Wood Anatomists Committee. (1989). IAWA List of Microscopic Features for Hardwood Identification. IAWA Bulletin n.s., 10(3), 219-332.

Johansen, D. A. (1940). Plant Microtechnique. Nueva York: McGraw-Hill.

López, L., \& Villalba, R. (2011). Climate Influences on the Radial Growth of Centrolobium microchaete, a Valuable Timber Species from the Tropical Dry Forests in Bolivia. BIOTROPICA, 43(1), 41-49.
Morales, M. S., Villalba, R., Grau H. R., Villagra, P. E., Boninsegna, J. A., Ripalta, A., \& Paolini, L. (2001). Potencialidad de Prosopis ferox Griseb (Leguminosae, subfamilia: Mimosoideae) para estudios dendrocronológicos en desiertos subtropicales de alta montaña. Revista Chilena de Historia Natural, 74(4), 865-872.

Morales, M. S., Villalba, R., Grau, H. R., \& Paolini, L. (2004). Rainfall-controlled tree growth in high-elevation subtropical treelines. Ecology, 85(11), 30803089. doi: 10.1890/04-0139

Morales, M. S., Christie, D. A., Villalba, R., Argollo, J., Pacajes, J., Silva, J. S., Alvarez, C. A., Llancabure, J. C., \& Solíz, C. (2012). Precipitation changes in the South American Altiplano since 1300 AD reconstructed by tree-rings. Climate of the Past, 8(2), 653-666. doi:10.5194/cp-8-653-2012

Oliveira, J. M. (2007). Anéis de crescimento de Araucaria angustifolia (Bertol.) O. Kuntze: bases de dendroecologia em ecosistemas subtropicais montanos no Brasil. (Doctoral thesis). Universidade Federal do Rio Grande do Sul, Porto Alegre.

Oliveira, J. M., Santarosa, E., Pillar, V. D., \& Roig, F. A. (2009). Seasonal cambium activity in the subtropical rain forest tree Araucaria angustifolia. Trees, 23(1), 107-115.

Oficina Nacional de Evaluación de Recursos Naturales. (1976). Mapa Ecológico del Perú. Guía Explicativa. Lima.

Priya, P. B., \& Bhat, K. M. (1998). False ring formation in teak (Tectona grandis Lf) and the influence of environmental factors. Forest Ecology and Management, $108(3), 215-222$.

Ramírez, J. A., \& Del Valle, J. I. 2012. Local and global climate signals from tree rings of Parkinsonia praecox in La Guajira, Colombia. International Journal of Climatology, 32 (7), 1077-1088. doi: 10.1002/ joc. 2335

Reátegui, F., \& Martínez, P. (2011). Evaluación forestal. (Informe temático). Iquitos: Proyecto Mesozonificación Ecológica y Económica para el Desarrollo Sostenible de la Provincia de Satipo, convenio entre el Instituto de Investigaciones de la Amazonía Peruana, Comisión Nacional para el Desarrollo y Vida sin Drogas y la Municipalidad Provincial de Satipo.

Rodríguez, R., Mabres, A., Luckman, B. H., Evans, M., Masiokas, M., \& Ektvedt, T. M. (2005). "El Niño" events recorded in dry-forest species of the lowlands of northwest Peru. Dendrochronologia, 22(3), 181-186.

Rodríguez, E. (2011). Clima (Informe temático). Iquitos: Proyecto Mesozonificación Ecológica y Económica para el Desarrollo Sostenible de la Provincia de Satipo, convenio entre el Instituto de Investigaciones de la Amazonía Peruana, Comisión Nacional para 
el Desarrollo y Vida sin Drogas y la Municipalidad Provincial de Satipo.

Roig, F. A. (Comp.). (2000). Dendrocronología en América Latina. Mendoza: Editorial de la Universidad Nacional de Cuyo.

Rosero, J. (2009). Dendrocronologia de árvores de mogno, Swietenia macrophylla King., Meliaceae, ocorrentesna floresta tropical Amazônica do Departamento de Madre de Dios, Perú. (Tesis de Maestría). Universidade de São Paulo, Piracicaba.

Solíz, C., Villalba, R., Argollo, J., Morales, M. S., Christie, D. A., Moya, J., \& Pacajes, J. (2009). Spatial and temporal variations in Polylepis tarapacana annual growth across the Bolivian Altiplano $\left(17-23^{\circ} \mathrm{S}\right)$. Palaeogeography Palaeoclimatology Palaeoecology, 281(3-4), 296-308.

Schöngart, J., Piedade, M. T. F., Ludwigshausen, S., \& Worbes, M. (2002). Phenology and stem-growth periodicity of tree species in Amazonian floodplain forests. Journal of Tropical Ecology, 18(4), 581-597. doi: http://dx.doi.org/10.1017/S0266467402002389

Schöngart, J., Junk, W. J., Piedade, M. T. F., Ayres, J. M., Hüttermann, A., \& Worbes, M. (2004). Teleconnection between tree growth in the Amazonian floodplains and the El Niño-Southern Oscillation effect. Global Change Biology 10(5), 683-692. doi: 10.1111/j.1529-8817.2003.00754.x

Schwyzer, A. (1988). Anillos de crecimiento en cuatro árboles tropicales de Jenaro Herrera. Loreto: Boletín de Lima 58.

Servicio Nacional de Meteorología e Hidrología del Perú. (2010). Base de datos de precipitación total mensual $(\mathrm{mm})$ y temperatura media mensual de la estación meteorológica de Satipo [CD-ROM]. Lima. SENAMHI.
Stokes, M. A., \& Smiley, T. L. (1968). An introduction to tree-ring dating. Illinois: The University of Press.

Valencia, G. M. (2011). Caracterización anatómica de anillos de crecimiento de especies forestales tropicalesSelva Central. (Tesis de Ingeniero). Universidad Nacional del Centro del Perú, Huancayo.

Vetter, R. E., \& Botosso, P. C. (1989). Remarks on age and growth rate determination of Amazonian trees. IAWA Bulletin n.s., 10, 133-145.

Villalba, R., Grau, H. R., Boninsegna, J. A., Jacoby, G. C., \& Ripalta, A. (1998). Tree-ring evidence for longterm precipitation changes in subtropical South America. International Journal of Climatology, 18(13), 1463-1478.

Villalba, R. (2000). Dendroclimatology: a southern hemisphere perspective. In P.P. Smolka \& W. Volkheimer (Eds.), Southern hemisphere paleo- and neoclimates. (pp. 27-57). Berlin: Springer Verlag.

Worbes, M. (1985). Structural and other adaptations to long-term flooding by trees in Central Amazonia. Amazoniana, 9(3), 459-484.

Worbes, M. (1989). Growth rings, increment and age of trees in inundation forests, savannas and a mountain forest in the Neotropics. IAWA Bulletin n.s., 10, 109-122.

Worbes, M. (1995). How to measure growth dynamics in tropical trees a review. Iawa Journal, 16(4), 337-351.

Worbes, M. (1999). Annual growth rings, rainfall-dependent growth and long-term growth patterns of tropical trees from the Caparo Forest Reserve in Venezuela. Journal of Ecology, 87(3), 391-403.

Worbes, M. (2001). Forest growth in the tropics. Freiburg.

Worbes, M. (2002). One hundred years of tree-ring research in the tropics-a brief history and an outlook to future challenges. Dendrochronologia, 20(1-2), 217-231. 
